\title{
Extremal Values of the Chromatic Number for a Given Degree Sequence
}

\author{
Stéphane Bessy ${ }^{1}$ and Dieter Rautenbach ${ }^{2}$
}

${ }^{1}$ Laboratoire d'Informatique, de Robotique et de Microélectronique de Montpellier (LIRMM), Montpellier, France, stephane.bessy@lirmm.fr

${ }^{2}$ Institute of Optimization and Operations Research, Ulm University, Ulm, Germany, dieter.rautenbach@uni-ulm.de

\begin{abstract}
For a degree sequence $d: d_{1} \geq \cdots \geq d_{n}$, we consider the smallest chromatic number $\chi_{\min }(d)$ and the largest chromatic number $\chi_{\max }(d)$ among all graphs with degree sequence $d$. We show that if $d_{n} \geq 1$, then $\chi_{\min }(d) \leq \max \left\{3, d_{1}-\frac{n+1}{4 d_{1}}+4\right\}$, and, if $\sqrt{n+\frac{1}{4}}-\frac{1}{2}>$ $d_{1} \geq d_{n} \geq 1$, then $\chi_{\max }(d)=\max _{i \in[n]} \min \left\{i, d_{i}+1\right\}$. For a given degree sequence $d$ with bounded entries, we show that $\chi_{\min }(d), \chi_{\max }(d)$, and also the smallest independence number $\alpha_{\min }(d)$ among all graphs with degree sequence $d$, can be determined in polynomial time.
\end{abstract}

Keywords: Degree sequence; chromatic number; independence number

MSC 2010: 05C07; 05C15; 05C69 


\section{Introduction}

We consider finite, simple, and undirected graphs. The degree sequence of a graph $G$ with vertex set $\left\{v_{1}, \ldots, v_{n}\right\}$ is the sequence $d_{G}\left(v_{1}\right), \ldots, d_{G}\left(v_{n}\right)$ of its vertex degrees. A sequence $d_{1}, \ldots, d_{n}$ of integers is a degree sequence if it is the degree sequence of some graph. Repetitions within the degree sequence can be indicated by suitable exponents; the degree sequence of the star $K_{1, r}$ of order $r+1$, for instance, is $r, 1^{r}$. For a given sequence $d$, let $\mathcal{G}(d)$ be the set of all graphs $G$ whose degree sequence is $d$; called the realizations of $d$. For an integer $n$, let $[n]$ be the set of the positive integers at most $n$.

In the present paper we consider

$$
\chi_{\min }(d)=\min \{\chi(G): G \in \mathcal{G}(d)\} \quad \text { and } \quad \chi_{\max }(d)=\max \{\chi(G): G \in \mathcal{G}(d)\} .
$$

Punnim [11] determined $\chi_{\min }(d)$ and $\chi_{\max }(d)$ for regular degree sequences $d=r^{n}$ in almost all cases. The parameter $\chi_{\max }(d)$ was also considered by Dvořák and Mohar [3], who established degree sequence versions of the Hadwiger Conjecture and even the Hajós Conjecture, see also 14].

We contribute some bounds, exact values, and algorithmic results. Further discussion of related research will be given throughout the rest of the paper.

\section{Some bounds and exact values}

For a sequence $d$ of non-negative integers $d_{1} \geq \cdots \geq d_{n}$, let $H(d)$ be the sequence

$$
d_{2}-1, \ldots, d_{d_{1}+1}-1, d_{d_{1}+2}, \ldots, d_{n}
$$

Havel [9] and Hakimi [6] showed that $d$ is a degree sequence if and only if $H(d)$ is a degree sequence. In fact, they observed that if $d$ is a degree sequence, then there is a realization $G$ of $d$ in which the neighbours of a vertex of degree $d_{1}$ have degrees $d_{2}, \ldots, d_{d_{1}+1}$. Iteratively applying this observation to a given degree sequence yields a realization that tends to contain a large complete subgraph on the vertices of large degrees, that is, such a realization may be expected to have high chromatic number.

In order to obtain a realization with hopefully small chromatic number, one can apply Havel and Hakimi's observation to the complement. More precisely, for a degree sequence $d$ as above, the sequence $\bar{d}$ defined as

$$
n-1-d_{n} \geq \cdots \geq n-1-d_{1}
$$

is also a degree sequence; in fact, the graphs in $\mathcal{G}(\bar{d})$ are exactly the complements $\bar{G}$ of the graphs $G$ in $\mathcal{G}(d)$. Furthermore, by the above observation of Havel and Hakimi, $\bar{d}$ has a realization in which the neighbors of a vertex of the largest degree $n-1-d_{n}$ have degrees $n-1-d_{n-1}, \ldots, n-1-d_{d_{n}+1}$. Equivalently, as already observed by Kleitman and Wang [10] in a more general form, $d$ has a realization in which the neighbors of a vertex of the smallest 
degree $d_{n}$ have degrees $d_{1}, \ldots, d_{d_{n}}$. In summary, we obtain that $d$ is a degree sequence if and only if the sequence $\bar{H}(d)$ defined as

$$
d_{1}-1, \ldots, d_{d_{n}}-1, d_{d_{n}+1}, \ldots, d_{n-1}
$$

is a degree sequence. Iteratively applying this observation to a given degree sequence yields a realization that tends to avoid dense subgraphs on the vertices of large degrees, that is, such a realization may be expected to have small chromatic number.

As an example consider the degree sequence $d: r^{r+1}, 1^{r(r+1)}$ for some positive integer $r$. Havel and Hakimi's original observation yields the realization $K_{r+1} \cup\left(\begin{array}{l}r \\ 2\end{array}\right) K_{2}$, whose chromatic number is $r+1$, which equals $\chi_{\max }(d)$, while the above complementary version yields the realization $(r+1) K_{1, r}$, whose chromatic number is 2 , which equals $\chi_{\min }(d)$.

For a sequence $d$ of integers $d_{1}, \ldots, d_{n}$, let $n$ be the length of $d$, let $\min (d)=\min \left\{d_{1}, \ldots, d_{n}\right\}$, and let $\max (d)=\max \left\{d_{1}, \ldots, d_{n}\right\}$. Furthermore, let $\bar{H}^{0}(d)=d, \bar{H}^{1}(d)=\bar{H}(d)$, and $\bar{H}^{i}(d)=$ $\bar{H}\left(\bar{H}^{i-1}(d)\right)$ for an integer $i$ at least 2 . Note that iteratively applying the reductions $d \mapsto H(d)$ or $d \mapsto \bar{H}(d)$ always requires reordering the constructed sequences in a non-increasing way.

Theorem 1 If $d$ is a degree sequence of length $n$, then

$$
\chi_{\min }(d) \leq \max \left\{\min \left(\bar{H}^{n-i}(d)\right): i \in[n]\right\}+1
$$

Proof: Iteratively applying the complementary version of Havel and Hakimi's observation to the degree sequence $d$ yields a realization $G$ of $d$ with vertex set $\left\{v_{1}, \ldots, v_{n}\right\}$ such that, for $i$ from $n$ down to 1 , the vertex $v_{i}$ has degree $\min \left(\bar{H}^{n-i}(d)\right)$ in the graph $G\left[\left\{v_{1}, \ldots, v_{i}\right\}\right]$. Greedily coloring the vertices of $G$ in the order $v_{1}, \ldots, v_{n}$ yields a coloring that uses at most $\max \left\{\min \left(\bar{H}^{n-i}(d)\right): i \in[n]\right\}+1$ colors.

Note that for the degree sequence $d: r^{r+1}, 1^{r(r+1)}$ of length $n=(r+1)^{2}$ considered as an example above, we obtain $\max \left\{\min \left(\bar{H}^{n-i}\left(r^{r+1}, 1^{r(r+1)}\right)\right): i \in[n]\right\}+1=2$, that is, for this degree sequence $d$, Theorem 1 reproduces the correct value of $\chi_{\min }(d)$.

Unfortunately, Theorem 1 is not very explicit. As a more explicit consequence, we quantify how small degrees may reduce the effect of large degrees on $\chi_{\min }(d)$.

Corollary 2 If $d$ is a degree sequence $d_{1} \geq \ldots \geq d_{n}$, and $k$ and $\ell$ are positive integers such that $d_{k} \geq k+\ell$ and $d_{n-\ell+1} \leq k$, then

$$
\chi_{\min }(d) \leq \max \left\{d_{1}-\frac{1}{k}\left(1+\sum_{i=n-\ell+1}^{n} d_{i}\right)+1, d_{k+1}, k\right\}+1
$$

Proof: We consider the first $\ell$ applications of the reduction $d \mapsto \bar{H}(d)$. Since $d_{k} \geq k+\ell$ and $d_{n-\ell+1} \leq k$, we obtain that, for $i \in[\ell]$, the degree sequence $\bar{H}^{i}(d)$ arises from $\bar{H}^{i-1}(d)$ by removing the degree $d_{n-i+1}$, and reducing the $d_{n-i+1}$ largest degrees by 1 . For $i \in\{0, \ldots, \ell\}$, let $\Delta_{i}=\max \left(\bar{H}^{i}(d)\right)$, and let $n_{i}$ be the number of entries of $\bar{H}^{i}(d)$ that are equal to $\Delta_{i}$. Suppose, 
for a contradiction, that $\Delta_{\ell}>\max \left\{d_{1}-\frac{D+1}{k}+1, d_{k+1}\right\}$, where $D=\sum_{i=n-\ell+1}^{n} d_{i}$. Note that each of the $\ell+1$ degree sequences $d, \bar{H}(d), \ldots, \bar{H}^{\ell}(d)$ contains at most $k$ entries that are strictly larger than $d_{k+1}$. So, for $i \in[\ell]$, we have

- $\left(\Delta_{i}, n_{i}\right)=\left(\Delta_{i-1}, n_{i-1}-d_{n-i+1}\right)$ if $d_{n-i+1}<n_{i-1}$, and

- $\Delta_{i}=\Delta_{i-1}-1$ and $n_{i} \leq k-\left(d_{n-i+1}-n_{i-1}\right)=n_{i-1}-d_{n-i+1}+k$ if $d_{n-i+1} \geq n_{i-1}$.

Note that $\left(k \Delta_{i-1}+n_{i-1}\right)-\left(k \Delta_{i}+n_{i}\right) \geq d_{n-i+1}$ in both cases. Summation over $i \in[\ell]$ yields $\left(k \Delta_{0}+n_{0}\right)-\left(k \Delta_{\ell}+n_{\ell}\right) \geq D$. Since $\Delta_{0}=d_{1}, n_{0} \leq k$, and $n_{\ell} \geq 1$, this implies $\Delta_{\ell} \leq d_{1}-\frac{D+1}{k}+1$, which is a contradiction. Hence, $\Delta_{\ell} \leq \max \left\{d_{1}-\frac{D+1}{k}+1, d_{k+1}\right\}$, and any realization $H$ of the degree sequence $\bar{H}^{\ell}(d)$ can be colored using at most $\max \left\{d_{1}-\frac{D+1}{k}+1, d_{k+1}\right\}+1$ many colors. Adding $\ell$ further vertices of degrees $d_{n-\ell+1}, \ldots, d_{n}$ one by one to $H$, and connecting them to suitable vertices according to the previous reductions, yields a realization $G$ of $d$. Since the added vertices all have degree at most $k$, the coloring of $H$ can be extended greedily to a coloring of $G$ using at most $\max \left\{d_{1}-\frac{D+1}{k}+1, d_{k+1}, k\right\}+1$ different colors in total.

For a given degree sequence $d$ not satisfying any further restriction, one can only bound $\chi_{\min }(d)$ from above by $\max (d)+1$. In fact, $d$ might be $\max (d)^{\max (d)+1}, 0^{n-\max (d)-1}$, whose only realization contains a clique of size $\max (d)+1$.

Our next two results improve this trivial estimate for graphs without isolated vertices.

Theorem 3 If $d$ is a degree sequence of length $n$ with $\max (d) \geq \sqrt{\frac{n \delta}{4}}$ and $\min (d) \geq \delta$ for some positive integer $\delta$, then $\chi_{\min }(d) \leq \max (d)-\frac{n \delta}{4 \max (d)}+\delta+3$.

Proof: Our first goal is to show that we may assume that $d$ has a realization with a very large independent set. Therefore, among all realizations $G$ of the degree sequence $d$ and all (not necessarily optimal) colorings $f$ of $G$, we choose $G$ and $f$ with color classes $V_{1}, \ldots, V_{k}$, where $V_{i}$ contains $n_{i}$ vertices for $i \in[k]$, in such a way that

- $\left(n_{1}, \ldots, n_{k}\right)$ is lexicographically maximal, and

- subject to this first condition, the number of edges between $V_{k-1}$ and $V_{k}$ is minimum.

Note that $k$ may actually be larger than $\chi(G)$, and that $n_{1}$ is necessarily equal to the independence number $\alpha(G)$ of $G$.

Let $\Delta=\max (d)$. If $k \leq \Delta-\frac{n \delta}{4 \Delta}+\delta+3$, then $\chi_{\min }(d) \leq \chi(G) \leq k$ implies the desired bound. Hence, we may assume that $k>\Delta-\frac{n \delta}{4 \Delta}+\delta+3$. Since $\Delta \geq \sqrt{\frac{n \delta}{4}}$ and $\delta \geq 1$, we have $k \geq 5$. By the choice of the coloring $f$, there is an edge, say $u v$, between the smallest two color classes $V_{k-1}$ and $V_{k}$. If $G \backslash\left(V_{k-1} \cup V_{k} \cup N_{G}(u) \cup N_{G}(v)\right)$ contains an edge $x y$, then removing from $G$ the two edges $u v$ and $x y$, and adding the two edges $u x$ and $v y$, yields another realization $G^{\prime}$ of $d$. Note that $f$ is still a coloring of $G^{\prime}$. This implies that there is a coloring $f^{\prime}$ of $G^{\prime}$ such that either the non-increasing vector of the sizes of the color classes is lexicographically larger than the one of $f$, or there are fewer edges between the two smallest color classes. Since both cases imply a 
contradiction to the choice of $G$ and $f$, we obtain that $V(G) \backslash\left(V_{k-1} \cup V_{k} \cup N_{G}(u) \cup N_{G}(v)\right)$ is an independent set, which implies $\alpha(G) \geq n-\left(n_{k-1}+n_{k}\right)-2 \Delta$. Since $V_{k-1}$ and $V_{k}$ are the smallest two color classes, and $n_{2}+\cdots+n_{k}=n-\alpha(G)$, we obtain $n_{k-1}+n_{k} \leq \frac{2}{k-1}(n-\alpha(G))$. This implies $\alpha(G) \geq n-\frac{2}{k-1}(n-\alpha(G))-2 \Delta$, and, using $k \geq 5$, we obtain $\alpha(G) \geq n-\frac{k-1}{k-3} \cdot 2 \Delta \geq n-4 \Delta$.

Altogether, we may assume that $d$ has a realization $G$ with an independent set $I=$ $\left\{u_{1}, \ldots, u_{\alpha}\right\}$ of order at least $n-4 \Delta$. By the above-mentioned observations of Havel [9], Hakimi [6], Rao [12], and Kleitman and Wang [10], we may further assume that, for every $i \in[\alpha]$, the vertex $u_{i}$ is adjacent to $d_{G}\left(u_{i}\right)$ vertices in $V(G) \backslash I$ of the largest degrees in the induced subgraph $G-\left\{u_{1}, \ldots, u_{i-1}\right\}$ of $G$. Arguing as in the proof of Corollary 2, we obtain $((n-\alpha) \Delta+(n-\alpha))-((n-\alpha) \Delta(G-I)+1) \geq d_{G}\left(u_{1}\right)+\cdots+d_{G}\left(u_{\alpha}\right) \geq \alpha \delta$, where $\Delta(G-I)$ denotes the maximum degree of $G-I$. This implies $\Delta(G-I) \leq \Delta-\frac{\alpha \delta+1}{n-\alpha}+1 \leq$ $\Delta-\frac{(n-4 \Delta) \delta+1}{4 \Delta}+1=\Delta-\frac{n \delta+1}{4 \Delta}+\delta+1$. Therefore, we can color $G$ using at most $\Delta-\frac{n \delta+1}{4 \Delta}+\delta+2$ colors on the vertices in $V(G) \backslash I$, and one additional color on the vertices in $I$, which implies $\chi_{\min }(d) \leq \chi(G) \leq \Delta-\frac{n \delta+1}{4 \Delta}+\delta+3$.

For positive integers $r, s$, and $\delta$ such that $r+1$ is a multiple of $\delta$, let $d$ be the degree sequence $(r+s)^{r+1}, \delta^{s(r+1) / \delta}$. Since the sum of the largest $r+1$ degrees equals exactly $2\left(\begin{array}{c}r+1 \\ 2\end{array}\right)+\delta s(r+1) / \delta$, every realization $G$ of $d$ contains a clique on the $r+1$ vertices of largest degrees, and an independent set on the remaining vertices. Note that $\chi(G) \in\{r+1, r+2\}$, which, for $r \gg s \gg \delta$, is roughly $\max (d)-\frac{n \min (d)}{\max (d)}$, that is, up to the constants, the bound in Theorem 3 is best possible. In fact, by imposing a stronger lower bound on $\max (d)$ or by increasing the additive constant, the factor 4 within the term $\frac{n \delta+1}{4 \Delta}$ can easily be reduced to slightly more than 2 .

Our next result gives a best possible bound on $\chi_{\min }(d)$ for degree sequences of small degrees.

Theorem 4 If $n, d_{1}, \ldots, d_{n}$ are integers such that $\sqrt{\frac{n-1}{2}} \geq d_{1} \geq \cdots \geq d_{n} \geq 1$ and $d_{1}+\cdots+d_{n}$ is even, then $\chi_{\min }(d) \leq 3$. (In particular, $d_{1}, \ldots, d_{n}$ is a degree sequence.)

Proof: There is a partition of $[n]$ into two sets $X$ and $Y$ with ||$X|-| Y|| \leq 1$ and $0 \leq s \leq$ $d_{1} \leq \sqrt{\frac{n-1}{2}}$, where $s=\sum_{i \in X} d_{i}-\sum_{i \in Y} d_{i}$; in fact, as long as there are two equal entries $d_{i}$ and $d_{j}$ in the sequence $d_{1}, \ldots, d_{n}$, we assign $i$ to $X$ and $j$ to $Y$, and remove $d_{i}$ and $d_{j}$ from the sequence, and once all remaining entries are distinct, say $d_{i_{1}}>\cdots>d_{i_{k}}$, we assign $i_{1}, i_{3}, \ldots$ to $X$ and $i_{2}, i_{4}, \ldots$ to $Y$. Let $x=|X|$ and $y=|Y|$. Note that $x, y \geq \frac{n-1}{2}$; in particular, $s \leq x$. Reducing $s$ distinct entries of the sequence $\left(d_{i}\right)_{i \in X}$ by 1 , and reordering yields a sequence $a_{1} \geq \cdots \geq a_{x}$. Reordering the sequence $\left(d_{i}\right)_{i \in Y}$ yields $b_{1} \geq \cdots \geq b_{y}$.

By construction, $\sum_{i \in[x]} a_{i}=\sum_{i \in[y]} b_{i}, \max \left\{a_{1}, b_{1}\right\} \leq \sqrt{\frac{n-1}{2}}$, and $b_{y} \geq 1$.

Let $k \in[x]$. If $k \leq \sqrt{\frac{n-1}{2}}$, then $a_{1} \leq \sqrt{\frac{n-1}{2}}$ and $b_{n} \geq 1$ imply

$$
\sum_{i \in[k]} a_{i} \leq k a_{1} \leq \frac{n-1}{2} \leq y \leq \sum_{i \in[y]} \min \left\{k, b_{i}\right\} .
$$


If $k>\sqrt{\frac{n-1}{2}}$, then $b_{1} \leq \sqrt{\frac{n-1}{2}}$ implies

$$
\sum_{i \in[k]} a_{i} \leq \sum_{i \in[x]} a_{i}=\sum_{i \in[y]} b_{i}=\sum_{i \in[y]} \min \left\{k, b_{i}\right\}
$$

By the Gale-Ryser Theorem [5, 15], there is a bipartite graph $H$ with partite sets $X$ and $Y$ with $|X|=x$ and $|Y|=y$ such that the vertices in $X$ have degrees $a_{1}, \ldots, a_{x}$ and the vertices in $Y$ have degrees $b_{1}, \ldots, b_{y}$. Since $s$ has the same parity as $\sum_{i \in X} d_{i}+\sum_{i \in Y} d_{i}=d_{1}+\cdots+d_{n}$, it is an even integer, and adding to $H$ a matching of size $s / 2$ incident to those vertices in $X$ corresponding to the entries of $\left(d_{i}\right)_{i \in X}$ that were previously reduced by 1 , results in a graph $G$ with degree sequence $d_{1}, \ldots, d_{n}$. Clearly, $\chi(G) \leq 3$, and the upper bound on $\chi_{\min }(d)$ follows.

The conclusion of Theorem 4 is best possible, because there might not be a subset $X$ of $[n]$ with $\sum_{i \in X} d_{i}=\sum_{i \in[n] \backslash X} d_{i}$, which is a necessary condition for the existence of a bipartite realization. The complexity of deciding the existence of a bipartite realization for a given degree sequence is unknown.

Note that together, Theorem 3 and Theorem 4 imply

$$
\chi_{\min }(d) \leq \max \left\{3, \max (d)-\frac{n+1}{4 \max (d)}+4\right\}
$$

for every degree sequence $d$ with $\min (d) \geq 1$.

Theorem 4 has the following variant where the essential assumption is that $\max (d)-\min (d)$ is small. Note that this next result also covers regular degree sequences of sufficient length.

Theorem 5 If $n, d_{1}, \ldots, d_{n}$ are integers and $\epsilon>0$ is such that $\frac{n-1}{2} \epsilon \geq d_{1} \geq \cdots \geq d_{n} \geq 1$, $d_{1}-d_{n} \leq \sqrt{\frac{n-1}{2}}(1-\epsilon)$, and $d_{1}+\cdots+d_{n}$ is even, then $\chi_{\min }(d) \leq 3$.

Proof: We may assume that $d_{1}>\sqrt{\frac{n-1}{2}}$; otherwise Theorem 4 implies the result. Furthermore, we have $\epsilon \leq 1$. Exactly as in the proof of Theorem 4, we obtain the existence of a partition of $[n]$ into two sets $X$ and $Y$ with ||$X|-| Y|| \leq 1$ and $0 \leq s \leq d_{1} \leq \frac{n-1}{2} \epsilon$, where $s=\sum_{i \in X} d_{i}-\sum_{i \in Y} d_{i}$. Setting $x=|X|$ and $y=|Y|$, we obtain, as above, that $x, y \geq \frac{n-1}{2}, s \leq x$, and $s$ is even. Let $a_{1} \geq \cdots \geq a_{x}$ and $b_{1} \geq \cdots \geq b_{y}$ be as in the proof of Theorem 4. By construction, $\sum_{i \in[x]} a_{i}=\sum_{i \in[y]} b_{i}, \max \left\{a_{1}, b_{1}\right\} \leq d_{1}$, and $b_{y} \geq d_{n}$.

Notice that as $d_{1}>\sqrt{\frac{n-1}{2}}$, we have

$$
\frac{d_{n}}{d_{1}} \geq \frac{d_{1}-\sqrt{\frac{n-1}{2}}(1-\epsilon)}{d_{1}} \geq 1-(1-\epsilon)=\epsilon .
$$

Let $k \in[x]$. If $k \leq d_{n}$, then

$$
\sum_{i \in[k]} a_{i} \leq k d_{1} \leq k \frac{n-1}{2} \leq k y \leq \sum_{i \in[y]} \min \left\{k, b_{i}\right\} .
$$


If $d_{n}<k<d_{1}$, then

$$
\sum_{i \in[k]} a_{i} \leq k d_{1} \leq d_{1}^{2} \leq \frac{n-1}{2} \epsilon d_{1} \leq \frac{n-1}{2} d_{n} \leq y d_{n} \leq \sum_{i \in[y]} \min \left\{k, b_{i}\right\}
$$

And, if $k \geq d_{1}$, then

$$
\sum_{i \in[k]} a_{i} \leq \sum_{i \in[x]} a_{i}=\sum_{i \in[y]} b_{i}=\sum_{i \in[y]} \min \left\{k, b_{i}\right\}
$$

At this point, the proof can be completed exactly as the proof of Theorem 4 ,

For a graph $G$ with degree sequence $d_{1} \geq \cdots \geq d_{n}$, Welsh and Powell [16] observed

$$
\chi(G) \leq \max _{i \in[n]} \min \left\{i, d_{i}+1\right\}
$$

which is an immediate consequence of applying the natural greedy coloring algorithm to the vertices of $G$ in an order of non-increasing degrees. If $d_{1} \geq \cdots \geq d_{n}$ is a degree sequence such that $d_{p}-d_{p+1} \geq p-2$ for $p=\max _{i \in[n]} \min \left\{i, d_{i}+1\right\}$, then Havel and Hakimi's observation explained above implies the existence of a realization $G$ of $d$ for which the vertices of degrees $d_{1}, \ldots, d_{p}$ form a clique. This implies $p \leq \chi(G) \leq \chi_{\max }(d) \leq p$, that is, $\chi_{\max }(d)=\max _{i \in[n]} \min \left\{i, d_{i}+1\right\}$ for such degree sequences.

Our next result shows that the Welsh-Powell bound (2) also gives the correct value of $\chi_{\max }(d)$ for degree sequences $d$ of small degrees.

Theorem 6 If $n, d_{1}, \ldots, d_{n}$ are integers such that $\sqrt{n+\frac{1}{4}}-\frac{1}{2}>d_{1} \geq \cdots \geq d_{n} \geq 1$ and $d_{1}+\cdots+d_{n}$ is even, then $\chi_{\max }(d)=\max _{i \in[n]} \min \left\{i, d_{i}+1\right\}$.

Proof: Let $p=\max _{i \in[n]} \min \left\{i, d_{i}+1\right\}$. Note that $p \leq d_{p}+1 \leq d_{1}+1$.

By the Welsh-Powell bound (2), every graph $G$ with degree sequence $d_{1}, \ldots, d_{n}$ satisfies $\chi(G) \leq p$, which implies $\chi_{\max }(d) \leq p$. In order to establish equality, we show the existence of a realization that contains a clique of size $p$.

Let $k \in[n]$. We obtain $\sum_{i \in[k]} d_{i} \leq k d_{1}$ and $k(k-1)+\sum_{i \in[n] \backslash[k]} \min \left\{k, d_{i}\right\} \geq k(k-1)+n-k$. Therefore, $\sum_{i \in[k]} d_{i}$ is at most $k(k-1)+\sum_{i \in[n] \backslash[k]} \min \left\{k, d_{i}\right\}$ if $k d_{1} \leq k(k-1)+n-k$, which is equivalent to $k\left(d_{1}+2-k\right) \leq n$. Since $\sqrt{n+\frac{1}{4}}-\frac{1}{2}>d_{1} \geq 1$ implies $n \geq 3$ and $k\left(d_{1}+2-k\right) \leq$ $\left(\frac{d_{1}+2}{2}\right)^{2} \leq n$, the Erdős-Gallai Theorem [4] implies the existence of a graph with degree sequence $d_{1}, \ldots, d_{n}$. Among all such graphs with vertex set $\left\{v_{1}, \ldots, v_{n}\right\}$, where $v_{i}$ has degree $d_{i}$ for $i \in[n]$, we choose $G$ such that the number $m\left(G\left[\left\{v_{1}, \ldots, v_{p}\right\}\right]\right)$ of edges of the subgraph of $G$ induced by $\left\{v_{1}, \ldots, v_{p}\right\}$ is as large as possible.

Suppose, for a contradiction, that $G\left[\left\{v_{1}, \ldots, v_{p}\right\}\right]$ is not a clique, that is, $v_{i}$ and $v_{j}$ are not adjacent in $G$ for distinct $i$ and $j$ in $[p]$. By the choice of $p$, we have $d_{i}, d_{j} \geq p-1$, which implies that $v_{i}$ and $v_{j}$ both have at least one neighbor in $R=\left\{v_{p+1}, \ldots, v_{n}\right\}$.

First, we assume that $v_{i}$ and $v_{j}$ both have the same unique neighbor $v_{r}$ in $R$, that is, $\left\{v_{r}\right\}=N_{G}\left(v_{i}\right) \cap R=N_{G}\left(v_{j}\right) \cap R$. Since there are at most $1+d_{1}^{2}$ vertices at distance at most 2 
from $v_{r}$, including, in particular, $v_{i}$ and $v_{j}$, and $n-(p-2)-\left(1+d_{1}^{2}\right) \geq n-d_{1}^{2}-d_{1}>0$, there is a vertex $v_{s}$ in $R$ with a neighbor $v_{t}$ such that $v_{s}$ and $v_{t}$ are both not adjacent to $v_{r}$. Now, removing from $G$ the edges $v_{i} v_{r}, v_{j} v_{r}$, and $v_{s} v_{t}$, and adding the edges $v_{i} v_{j}, v_{r} v_{s}$, and $v_{r} v_{t}$ yields a realization $G^{\prime}$ of $d_{1}, \ldots, d_{n}$ with $m\left(G^{\prime}\left[\left\{v_{1}, \ldots, v_{p}\right\}\right]\right)>m\left(G\left[\left\{v_{1}, \ldots, v_{p}\right\}\right]\right)$, which contradicts the choice of $G$.

Now, we may assume that $v_{i}$ is adjacent to some vertex $v_{r}$ in $R$, and that $v_{j}$ is adjacent to a different vertex $v_{s}$ in $R$. If $v_{r}$ is not adjacent to $v_{s}$, then removing from $G$ the edges $v_{i} v_{r}$ and $v_{j} v_{s}$, and adding the edges $v_{i} v_{j}$ and $v_{r} v_{s}$ yields a realization $G^{\prime}$ of $d_{1}, \ldots, d_{n}$ with $m\left(G^{\prime}\left[\left\{v_{1}, \ldots, v_{p}\right\}\right]\right)>m\left(G\left[\left\{v_{1}, \ldots, v_{p}\right\}\right]\right)$, which contradicts the choice of $G$. Hence, we may assume that $v_{r}$ and $v_{s}$ are adjacent. Since there are at most $1+d_{1}^{2}$ vertices at distance at most 2 from $v_{r}$, including, in particular, $v_{i}, v_{s}$, and $v_{j}$, and $n-(p-2)-\left(1+d_{1}^{2}\right) \geq n-d_{1}^{2}-d_{1}>0$, there is a vertex $v_{p}$ in $R$ with a neighbor $v_{q}$ such that $v_{p}$ is not adjacent to $v_{s}$, and $v_{q}$ is not adjacent to $v_{r}$. Note that $v_{q}$ may be $v_{j}$, in which case, $v_{j}$ has distance 2 from $v_{r}$. Now, removing from $G$ the edges $v_{i} v_{r}, v_{j} v_{s}$, and $v_{p} v_{q}$, and adding the edges $v_{i} v_{j}, v_{s} v_{p}$, and $v_{r} v_{q}$ yields a realization $G^{\prime}$ of $d_{1}, \ldots, d_{n}$ with $m\left(G^{\prime}\left[\left\{v_{1}, \ldots, v_{p}\right\}\right]\right)>m\left(G\left[\left\{v_{1}, \ldots, v_{p}\right\}\right]\right)$, which contradicts the choice of $G$.

Altogether, we obtain that $G$ contains a clique of order $p$, which completes the proof.

\section{Algorithmic aspects}

One way to establish that $\chi_{\max }(d)$ is large is to show the existence of a realization of $d$ that contains a large clique. Dvořák and Mohar [3] proved the best possible statement that for every degree sequence $d$, some realization of $d$ has a clique of size at least $5 / 6\left(\chi_{\max }(d)-3 / 5\right)$. Since Rao [12,13] efficiently characterized the largest clique size $\omega_{\max }(d)$ of any realization of a given degree sequence $d$, and, trivially, $\chi_{\max }(d) \geq \omega_{\max }(d)$, we immediately obtain that $\chi_{\max }(d)$ can be approximated in polynomial time for a given $d$ within an asymptotic factor of 6/5.

Our next two results show that $\chi_{\max }(d)$ and $\chi_{\min }(d)$ can both be determined in polynomial time for given degree sequences with bounded entries.

Corollary 7 Let $\Delta$ be a fixed positive integer.

For a given degree sequence $d$ with $\max (d) \leq \Delta$, one can determine $\chi_{\max }(d)$ in polynomial time.

Proof: Let $d$ have length $n$. Clearly, we may assume $\min (d) \geq 1$. If $\sqrt{n-2} \geq \Delta$, then Theorem 6 implies that $\chi_{\max }(d)$ coincides with the Welsh-Powell bound (2). If $\sqrt{n-2}<\Delta$, then, as $\Delta$ is fixed, there are only constantly many realizations of $d$, which can all be generated and optimally colored by brute force in constant time.

Theorem 8 Let $k$ and $p$ be fixed positive integers.

For a given degree sequence $d$ with at most $p$ distinct entries, one can decide in polynomial time whether $\chi_{\min }(d) \leq k$. 
Proof: Let $d: d_{1}^{n_{1}}, \ldots, d_{p}^{n_{p}}$ and $n=n_{1}+\cdots+n_{p}$. There are $\prod_{i=1}^{p}\left(\begin{array}{c}n_{i}+k-1 \\ k-1\end{array}\right) \leq\left(\frac{n}{p}+k\right)^{k p}$ distinct matrices $\left(n_{i}^{j}\right)_{(i, j) \in[p] \times[k]}$ with non-negative integral entries $n_{i}^{j}$ such that $\sum_{j=1}^{k} n_{i}^{j}=n_{i}$ for $i \in[p]$. It is easy to see that $\chi_{\min }(d) \leq k$ if and only if there is such a matrix $\left(n_{i}^{j}\right)_{(i, j) \in[p] \times[k]}$ for which the complete $k$-partite graph whose $j$ th partite set $V_{j}$ has order $\sum_{i=1}^{p} n_{i}^{j}$ for $j \in[k]$, has a factor $G$ such that $V_{j}$ contains exactly $n_{i}^{j}$ vertices of degree $d_{i}$ in $G$ for every $i \in[p]$ and $j \in[k]$. Since the existence of such a factor can be decided in polynomial time using matching methods, and, for fixed $k$ and $p$, there are only polynomially many different suitable matrices, the desired statement follows.

It seems plausible to wonder whether $\chi_{\max }(d)$ is linked to $\alpha_{\min }(d)$, the minimum independence number of a realization of $d$. While $\alpha_{\max }(d)=\omega_{\max }(\bar{d})$ can be determined efficiently using the results of Rao [12, 13], Bauer, Hakimi, Kahl, and Schmeichel [1] conjectured that it is computationally hard to determine $\alpha_{\min }(d)$ for a given degree sequence $d$.

Our next goal is to show that also $\alpha_{\min }(d)$ can be determined in polynomial time for given degree sequences $d$ with bounded entries.

For a degree sequence $d_{1}, \ldots, d_{n}$, let $\alpha_{C W}(d)=\sum_{i=1}^{n} \frac{1}{d_{i}+1}$. Caro [2] and Wei [17] proved that $\alpha(G) \geq \alpha_{C W}(d)$ for every graph $G$ with degree sequence $d$. For a connected graph $G$ with degree sequence $d$, Harant and Rautenbach [7] showed $\alpha(G) \geq k \geq \sum_{u \in V(G)} \frac{1}{d_{G}(u)-f(u)+1}$, where $k$ is an integer, and, for every vertex $u$ of $G, f(u)$ is a non-negative integer at most $d_{G}(u)$ such that $\sum_{u \in V(G)} f(u) \geq 2(k-1)$. This improved an earlier result of Harant and Schiermeyer [8].

If $\alpha_{C W}(d) \geq 2$, then $k \geq \alpha_{C W}(d)$ implies $2(k-1) \geq k \geq \alpha_{C W}(d)$, and, hence,

$$
\begin{aligned}
\alpha(G) & \geq \sum_{u \in V(G)} \frac{1}{d_{G}(u)-f(u)+1} \\
& =\alpha_{C W}(d)+\sum_{u \in V(G)}\left(\frac{1}{d_{G}(u)-f(u)+1}-\frac{1}{d_{G}(u)+1}\right) \\
& \geq \alpha_{C W}(d)+\frac{1}{(\max (d)+1)^{2}} \sum_{u \in V(G)} f(u) \\
& \geq\left(1+\frac{1}{(\max (d)+1)^{2}}\right) \alpha_{C W}(d) .
\end{aligned}
$$

Theorem 9 Let $\Delta$ be a fixed positive integer.

For a given degree sequence $d$ with $\max (d) \leq \Delta$, every component of every realization $G$ of $d$ with $\alpha(G)=\alpha_{\min }(d)$ has order at most $\left((\Delta+1)^{3}+1\right)\left(\left(\frac{\Delta+2}{2}\right)^{2}+\left(\begin{array}{c}\Delta+1 \\ 2\end{array}\right)\right)$. In particular, one can determine $\alpha_{\min }(d)$ in polynomial time.

Proof: Let $d$ be a degree sequence with $\max (d) \leq \Delta$. Let $G$ be a realization of $d$ with $\alpha(G)=\alpha_{\min }(d)$. Suppose, for a contradiction, that some component $K$ of $G$ has order $n(K)$ more than the stated value. Let $R$ be a set of $\left(\frac{\Delta+2}{2}\right)^{2}$ vertices of $K$. For $i \in[\Delta]$, let $V_{i}$ be the set of vertices of degree $i$ in $V(K) \backslash R$, and let $n_{i}=\left|V_{i}\right|$. Let $p_{i}=\left\lfloor\frac{n_{i}}{i+1}\right\rfloor$, and let $S_{i}$ arise by removing 
$p_{i}(i+1)$ vertices from $V_{i}$ for each $i \in[\Delta]$. Note that $|S| \leq \sum_{i=1}^{\Delta} i=\left(\begin{array}{c}\Delta+1 \\ 2\end{array}\right)$, where $S=S_{1} \cup \cdots \cup S_{\Delta}$, that is, $R \cup S$ is a set of at least $\left(\frac{\Delta+2}{2}\right)^{2}$ and at most $\left(\frac{\Delta+2}{2}\right)^{2}+\left(\begin{array}{c}\Delta+1 \\ 2\end{array}\right)$ many vertices of $K$. Let $d^{\prime}$ be the sequence of the degrees of the vertices in $R \cup S$, and let $d^{\prime \prime}$ be the sequence of the degrees of the vertices in $V(K) \backslash(R \cup S)$. Note that $\alpha_{C W}\left(d^{\prime \prime}\right) \geq \frac{(n(K)-|R \cup S|)}{\Delta+1}$. Hence, the lower bound on $n(K)$ implies $\left(1+\frac{1}{(\Delta+1)^{2}}\right) \alpha_{C W}\left(d^{\prime \prime}\right)=\frac{1}{(\Delta+1)^{2}} \alpha_{C W}\left(d^{\prime \prime}\right)+\alpha_{C W}\left(d^{\prime \prime}\right)>|R \cup S|+\alpha_{C W}\left(d^{\prime \prime}\right)$. As observed in the proof of Theorem 6, the Erdős-Gallai Theorem implies that the sequence $d^{\prime}$, which is a sequence of positive integers at most $\Delta$ that is of length at least $\left(\frac{\Delta+2}{2}\right)^{2}$, is a degree sequence. Let $K_{0}^{\prime}$ be a realization of $d^{\prime}$. By construction, the graph $K^{\prime}=K_{0}^{\prime} \cup \bigcup_{i=1}^{\Delta} p_{i} K_{i+1}$ has exactly the same degree sequence as $K$. By the result of Harant and Rautenbach mentioned above,

$$
\begin{aligned}
\alpha\left(K^{\prime}\right) & =\alpha\left(K_{0}^{\prime}\right)+\sum_{i=1}^{\Delta} p_{i} \alpha\left(K_{i+1}\right) \\
& =\alpha\left(K_{0}^{\prime}\right)+\alpha_{C W}\left(d^{\prime \prime}\right) \\
& \leq|R \cup S|+\alpha_{C W}\left(d^{\prime \prime}\right) \\
& <\left(1+\frac{1}{(\Delta+1)^{2}}\right) \alpha_{C W}\left(d^{\prime \prime}\right) \\
& <\left(1+\frac{1}{(\Delta+1)^{2}}\right) \alpha_{C W}(d) \\
& \leq \alpha(K) .
\end{aligned}
$$

Therefore, replacing $K$ by $K^{\prime}$ within $G$ yields a realization $G^{\prime}$ of $d$ with $\alpha\left(G^{\prime}\right)<\alpha(G)$, contradicting the choice of $G$. This completes the proof of the first part of the statement.

Since, as $\Delta$ is fixed, there are only finitely many graphs of maximum degree at most $\Delta$ and order at most $\left((\Delta+1)^{3}+1\right)\left(\left(\frac{\Delta+2}{2}\right)^{2}+\left(\begin{array}{c}\Delta+1 \\ 2\end{array}\right)\right)$. Listing, for each of these graphs, the degree sequence and the independence number, it is a routine matter to determine $\alpha_{\min }(d)$ for a given degree sequence $d$ with $\max (d) \leq \Delta$ by dynamic programming in polynomial time.

\section{References}

[1] D. Bauer, S.L. Hakimi, N. Kahl, E. Schmeichel, Best Monotone Degree Bounds for Various Graph Parameters, Congressus Numerantium 192 (2008) 75-84.

[2] Y. Caro, New results on the independence number, Technical Report, Tel-Aviv University, 1979.

[3] Z. Dvořák, B. Mohar, Chromatic number and complete graph substructures for degree sequences, Combinatorica 33 (2013) 513-529.

[4] P. Erdős, T. Gallai, Graphs with prescribed degrees of vertices (Hungarian), Matematikai Lapok 11 (1960) 264-274. 
[5] D. Gale, A theorem on flows in networks, Pacific Journal of Mathematics 7 (1957) 1073-1082.

[6] S.L. Hakimi, On realizability of a set of integers as degrees of the vertices of a linear graph. I, Journal of the Society for Industrial and Applied Mathematics 10 (1962) 496-506.

[7] J. Harant, D. Rautenbach, Independence in connected graphs, Discrete Applied Mathematics 159 (2011) 79-86.

[8] J. Harant, I. Schiermeyer, On the independence number of a graph in terms of order and size, Discrete Mathematics 232 (2001) 131-138.

[9] V. Havel, A remark on the existence of finite graphs, Časopis Pro Pěstování Matematiky 80 (1955) 477-480.

[10] D.J. Kleitman, D.L. Wang, Algorithms for constructing graphs and digraphs with given valences and factors, Discrete Mathematics 6 (1973) 79-88.

[11] N. Punnim, Degree Sequences and Chromatic Numbers of Graphs, Graphs and Combinatorics 18 (2002) 597-603.

[12] R.A. Rao, The clique number of a graph with a given degree sequence, ISI Lecture Notes Series 4 (1979) 251-267.

[13] A.R. Rao, An Erdős-Gallai type result on the clique number of a realization of a degree sequence (unpublished).

[14] N. Robertson, Z.-X. Song, Hadwiger number and chromatic number for near regular degree sequences, Journal of Graph Theory 64 (2010) 175-183.

[15] H.J. Ryser, Combinatorial properties of matrices of zeros and ones, Canadian Journal of Mathematics 9 (1957) 371-377.

[16] D.J.A. Welsh, M.B. Powell, An upper bound for the chromatic number of a graph and its application to timetabling problems, The Computer Journal 10 (1967) 85-86.

[17] V.K. Wei, A lower bound on the stability number of a simple graph, Technical memorandum, TM 81 - 11217 - 9, Bell laboratories, 1981. 